(C) Owner/Author | ACM 2020. This is the author's version of the work. It is posted here for your personal use. Not for redistribution. The definitive Version of Record was published in ISS '20: Companion Proceedings of the 2020 Conference on Interactive Surfaces and Spaces https://doi.org/10.1145/3380867.3426204

\title{
Interactions with Digital Mountains: Tangible, Immersive and Touch Interactive Virtual Reality
}

\section{OSCAR ARDAIZ*}

Public University of Navarra

ASIER MARZO

Public University of Navarra

RUBEN BAZTAN

Public University of Navarra

IÑIGO EZCURDIA

Public University of Navarra

Digitization of Earth mountains and terrains has facilitated to plan journeys, manage natural resources, and learn about the Earth from the comfort of our homes. We aim to develop new interactions on digital mountains with novel interfaces: 3D printed representation of a mountain, an immersive virtual reality visualization, and two different touch interactive interfaces for immersive virtual reality visualizations: a 3D printed mountain with touch sensors and a multitouch tablet. We show how we have built such prototypes based on digital data retrieved from a map provider, and which interactions are possible with each interaction device. We explain how we design and conduct evaluation.

CCS CONCEPTS • Human-centered computing • Human computer interaction $(\mathrm{HCl}) \cdot$ Interaction paradigms

Additional Keywords and Phrases: Virtual Reality, 3D printing, Tangible interfaces, Digital mountain, Touch sensors.

ACM Reference Format:

\section{INTRODUCTION}

A digital mountain is the data that represents a mountain or any other terrain. Currently a digital mountain includes altitude data and images of the mountain surface. Digitization of mountains is a process performed with drones or satellites equipped with scanning sensors such as color cameras to obtain human-eye visible 
images, lidar and sonars to obtain distance to the sensor data. Different public and private organizations have carried out the digitization process. Those organizations publish and share such data to create better with larger coverage data sets, which are provided as data set or services over the Internet. We have chosen as our provider the company Mapbox since they provide high quality services and support.

Digital mountains data is used in Apps and Web applications by many types of users: mountaineers, and hikers use it to plan their journeys; architects, urban planners, mining companies, and others use them to plan and forecast dams, open mines, road and bridge pathways; educators and students use them to learn about natural resources and planet Earth.

Novel interaction technologies and devices will permit to visualize and interact with digital mountains in more intuitive and effective ways. The goal of our work is to build demonstrations of different interaction technologies with a digital mountain.

\section{RELATED WORK}

Research on interaction with digital mountains data can be classified into improvements to visualization techniques, and interactions through new devices. Novel visualization techniques explore how to control cameras [1] or modify terrain shearing to improve the perception of 3D data [4]. Novel interactions with digital mountains comprise augmented reality as a mean to visualize 3D maps over 2D maps [3], or tangible 3D digital mountains created with clay and images projections [2].

\section{DESIGN AND IMPLEMENTATION}

\subsection{Mountains in Virtual Reality}

Digital mountains can be re-created in a virtual representation where you can visualize the mountain in an immersive environment (Figure 1. Left). With the same data used to build 3D printed mountains, we have created a virtual reality visualization as a Unity program that is visualized with an Oculus head mounted display. In the virtual environment the viewfinder camera is placed at an altitude above the mountain to get a bird's eye view of the mountain. User turning their head results on the analogue movement on the virtual landscape, allowing the user to explore all the landscape from different point of views.

\subsection{Tangible 3D Printed Mountains}

We 3D print mountains in dimensions that can be hold with a hand (15x20 cm wide-length, 2 to $10 \mathrm{~cm}$ height) so that the user can visualize the topology of a mountain and touch to point locations to other users. Mountains are 3D printed with full color images of its surface for more realistic visualization (Figure 2. Left). We have created a process that generates a 3D printable object based on a user search. User inputs the name of a mountain, its geolocation and elevation data are retrieved to build a geometry on which we place satellite images to be $3 \mathrm{D}$ printed in full color. 


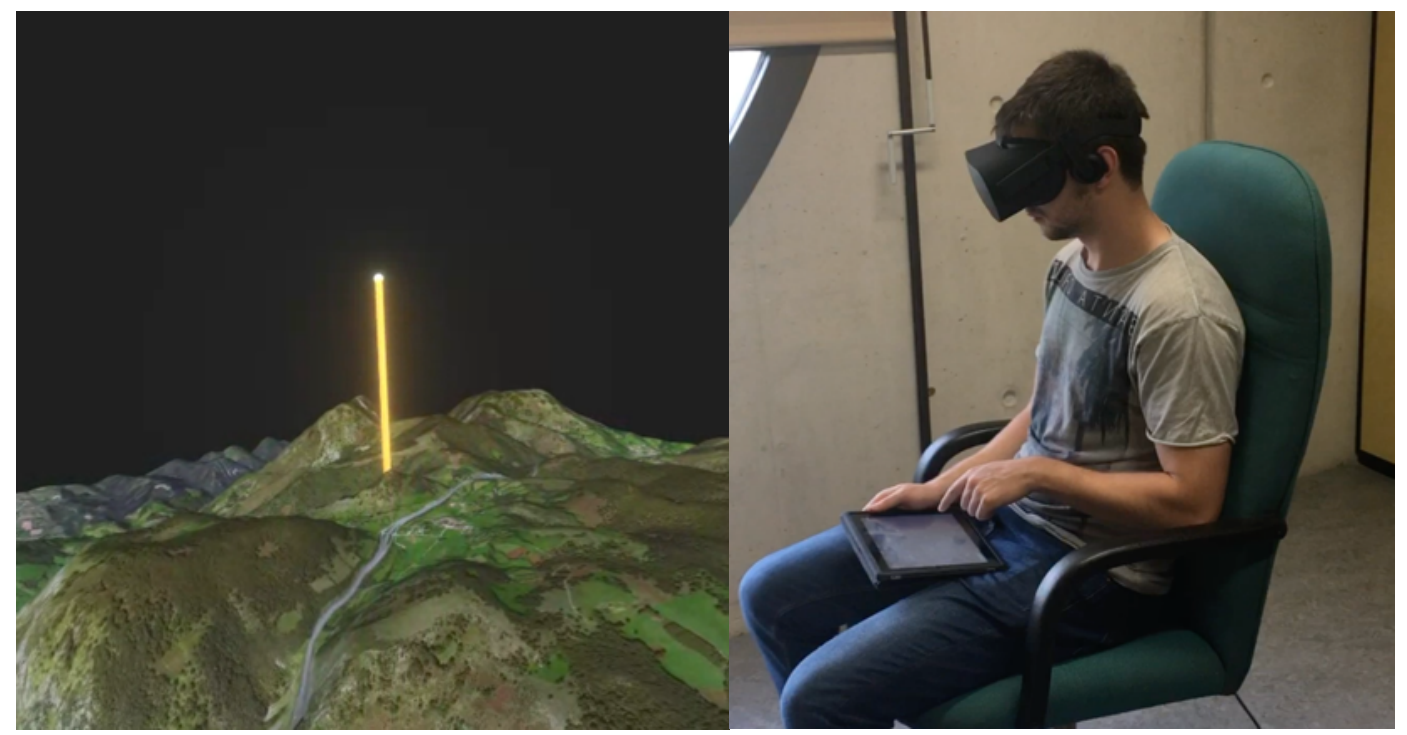

Figure 1: Left, immersive visualization of a mountain, a ray indicates an important waypoint location. Right, a user with a virtual reality head mounted display interacting with a virtual reality visualization through a tablet multitouch interface

\subsection{Touch Interactive Virtual Reality Mountains}

Interaction with virtual reality mountains is achieved with two mechanisms: touch sensors and mapping of generated signals to virtual reality interaction.

Touch-sensing is implemented as capacitive sensors. Capacitive sensors can be incorporated in a 3D printed object in two steps. Two-steps process require to 3D print the desired mountain with a hollow volume and to insert electronics and layer a patch of tin foil as near as possible to the surface desired to provide tactile interaction, this was the chosen option as it provided better touch sensitivity (Figure 2. Right).

We use an ESP32 microcontroller, which already incorporates 10 touch sensors and sends data through Wi-Fi Open Sound Control (OSC) protocol to the virtual reality computer. OSC is used to identify and route signals from multiple touch sensors and microcontrollers. The Unity-built virtual reality visualization receives signals from physical touch interfaces though a Unity third-party plugin: extOSC (https://assetstore.unity.com/packages/tools/input-management/extosc-open-sound-control-72005).

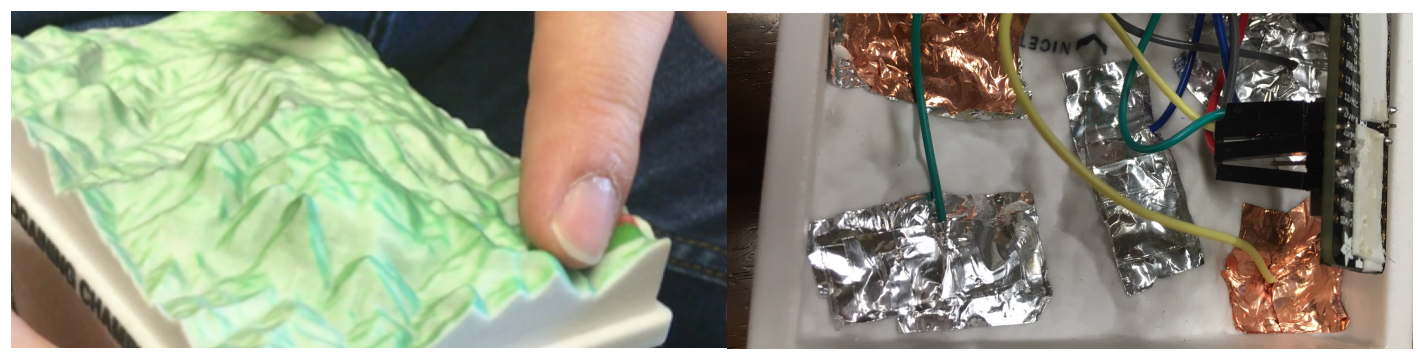

Figure 2: Left, 3D printed mountain user pointing to a peak; right, mountain is hollowed to contain electronics and sensors 


\section{INTERACTION USER STUDY}

Our goal is to study interactions afforded by the previous devices for intuitive and efficient user tasks. 3D printed digital mountains permit to visualize a miniature representation of a mountain with a tangible object, allowing to be physically shared with others for collaborative tasks. Virtual reality immersive visualizations permit a full scale $360^{\circ}$ visualization of a mountain from a birds' eye view or from any point in the surface. Interactions with a virtual reality immersive visualization performed with a 3D printed miniature representation will enable navigating the mountain with tactile references of the mountain topology. To complete the comparative study, we have also implemented a multitouch tablet interface that communicates with the virtual reality application using the same OSC protocol (Figure 1. Right). We performed a user study to evaluate visualizations and interactions while performing some common tasks with digital mountains: path planning and waypoint location. We summarize initial observations.

Regarding the 3D printed mountain miniature, we observed that collaboration was extremely natural: users pointed with their fingers to mountain locations and oriented the piece to provide the best viewing angle for their collaborators; the most commented disadvantage was the lack of text data about mountain names and waypoints.

Immersive visualization, on the contrary, did not allow any collaboration if only one user wore a head mounted display. However, users' viewing experience was more similar to reality when positioned over the surface.

The tablet multitouch interface was almost useless without a virtual pointer representation to transmit the user a sense of control (Fig 1. Left). When using 3D printed mountain as an interaction object, placing a virtual pointer is also required to locate touched point. This allows navigating through the virtual landscape by touching a distinguished part of the miniature: a high peak, a low valley or a distant steep slope. Despite of this, several users commented they could not relate the physical altitude variations to the virtual altitude variations as scales were too different.

Interaction with virtual reality mountains is achieved with two mechanisms: touch sensors and mapping of generated signals to virtual reality interaction.

\section{CONCLUSIONS}

Different interactions with digital mountains have been presented; each one has unique characteristics that are considered as very intuitive or efficient by users. However, the combination of an immersive virtual environment with a physical interaction element presents some difficulties. Perceiving touch-data without visual contact of the physical interaction device is hard and inaccurate.

\section{ACKNOWLEDGMENTS}

This work has been partially funded by the Government of Navarra research project PI043-2019.

\section{REFERENCES}

[1] Danyluk K., Jenny B., Willett W., Look-From Camera Control for 3D Terrain Maps, Proceedings of CHI '19.

[2] Piper B., Ratti C. and Ishii H. 2002. Illuminating Clay: A 3-D Tangible Interface for Landscape Analysis. Proceedings of CHI '02.

[3] Wiehr, F., Daiber, F., Kosmalla, F., \& Kr ${ }^{\circ}{ }^{\circ}$ ger, A. (2017). ARTopos: augmented reality terrain map visualization for collaborative route planning. UbiComp '17.

[4] Willett W., Jenny B., Isenberg T., Dragicevic P. Lightweight Relief Shearing for Enhanced Terrain Perception on Interactive Maps. Proceedings of CHI 2015), Apr 2015, Seoul, South Korea. 